\title{
EDITORIAL
}

\section{Airway mucus}

\author{
K.C. Kim*, P.K. Jeffery**
}

Hypersecretion of mucus is a cardinal sign of airways inflammation. Normally, secretion of mucus is designed to protect the respiratory portion of the lung from injury, but in disease its chronic overproduction in large airways (bronchi) and its appearance in small conducting airways (bronchioles) may be detrimental. Its relevance to patient discomfort and infection is generally accepted, but its relationship to rapid decline in lung function and death is much debated. The series "Airway mucus" represents a synopsis of part of the scientific programme of the annual congress of the Korean Society of Applied Pharmacology, held during April 1996, during which the views of an international forum of expert speakers were heard and discussed.

JEFFERY and Li [1] (London, UK) outline the structure and cellular origins of respiratory mucus, and present data on newly discovered mucin genes and the recent microscopic results of an endotoxin-induced animal model of mucous cell metaplasia and hypersecretion. The views of KIM [2], as a practising physician, are given in respect of the role and management of airway mucus in health and disease. The role of airway epithelium in inducing and modulating airway inflammation and its secretion of mucus via the production of proinflammatory cytokines are summarized by MARTIN and co-workers [3].

In vitro culture of airway epithelium is a requirement to enable us to begin to unravel the complexities and understand the many factors (e.g. dietary and interstitial), which determine its state of mucociliary differentiation. $\mathrm{WU}$ and co-workers [4] are internationally recognized workers, who provide us with an insight into these factors and remind us of the plasticity of epithelial cells. KIM and co-workers [5] review the structure and regulation of secretion of goblet cell mucins using primary airway epithelial cells in culture, which make it possible to investigate the biochemical mechanisms of secretion at a cellular level. WIDDICOMBE and co-workers [6] remind us that airway fluid is a mixture of mucus and the results of ion and fluid flux across both the surface and glandular epithelium: they review the factors

*Dept of Pharmaceutical Sciences, University of Maryland, Baltimore, USA. **Lung Pathology Unit, Imperial College at Royal Bromptom Hospital, London, UK. that regulate the depth and composition of surface liquid. The flow properties of mucus, which allow its normal clearance from the respiratory tract by mucociliary transport, and those properties which relate to the capacity to remove secretions by cough, are reviewed by KING [7], as are selected animal models which allow for the controlled study of mucous hypersecretion.

Finally, several classes of drug are capable of altering the secretion of mucus or its physical properties per se. These, together with the fascinating results of the use of Chinese traditional medicines, are presented by MiYata and Kai [8].

We hope that the review series "Airway mucus" will provide an informative update on an important topic of interest both to the scientist and clinician.

Acknowledgments: The authors wish to thank the members of the congress organizing commitee, especially Kwang Ho Ko and Young-Soo Shim (Seoul National University). The symposium was made possible by a generous grant from the Korean Green Cross Corporation. Sincere thanks are also due to the symposium presenters and chairmen.

\section{References}

1. Jeffery PK, Li D. Airway mucosa: secretory cells, mucus and mucin genes. Eur Respir J 1997; 10: 1655-1662.

2. Kim WD. Lung mucus: a clinician's view. Eur Respir $J 1997$ (in press).

3. Martin LD, Rochelle LG, Fischer BM, Krunkosky TM, Adler KB. Airway epithelium as an effector of secondary mediators. Eur Respir J 1997 (in press).

4. Wu R, Zhao YH, Chang MM. Growth and differentiation of conducting airway epithelial cells in culture. Eur Respir J 1997 (in press).

5. Kim KC, McCracken $\mathrm{K}$, Lee BC, et al. Airway goblet cell mucin. Its structure and regulation of secretion. Eur Respir J 1997 (in press).

6. Widdicombe J, Bastacky SJ, Wu DXY, Lee CY. Regulation of depth and composition of airway surface liquid. Eur Respir J 1997 (in press).

7. King M. Experimental models for studying mucocilliary clearance. Eur Respir J 1997 (in press).

8. Miyata T, Kai H. Current status of mucus drug research: strategies and problems. Eur Respir J 1997 (in press). 